\title{
Nanoscale Selective Passivation of Electrodes Contacting a 2D Semiconductor
}

\author{
Martina Lihter*, Michael Graf, Damir Iveković and Aleksandra Radenovic*
}

M. Lihter, Dr. M. Graf, Prof. A. Radenovic

Laboratory of Nanoscale Biology, Institute of Bioengineering, School of Engineering, EPFL, Lausanne, 1015, Switzerland

E-mail: martina.lihter@epfl.ch, aleksandra.radenovic@epfl.ch

Prof. D. Iveković

Laboratory of General and Inorganic Chemistry and Electroanalysis, Faculty of Food

Technology and Biotechnology, University of Zagreb, Zagreb, 10 000, Croatia

Keywords: electrodes, passivation, 2D semiconductors, molybdenum disulfide, poly(phenylene oxide), area selective

\begin{abstract}
Two-dimensional semiconducting materials have become the central component of various nanoelectronic devices and sensors. For sensors operating in liquid, it is crucial to efficiently block the electron transfer that occurs between the electrodes contacting the 2D material and the interfering redox species. This reduces current leakages and preserves a good signal-tonoise ratio. Here, we present a simple electrochemical method for passivating the electrodes contacting a monolayer of $\mathrm{MoS}_{2}$, a representative of transition metal dichalcogenide semiconductors. The method is based on blocking the electrode surface by a thin and compact layer of electronically non-conductive poly(phenylene oxide), PPO, formed by electrochemical polymerization of phenol. Since the phenol polymerization occurs in the potential window where $\mathrm{MoS}_{2}$ is electrochemically inactive, the PPO deposition is area-selective, limited to the electrode surface. The deposited PPO film is characterized by electrochemical, XPS, SEM, and AFM techniques. The applicability of this method is demonstrated by coating the electrodes of a $\mathrm{MoS}_{2}$-based FET coupled with a nanopore. The highly selective deposition, the simple approach, and the compatibility with $\mathrm{MoS}_{2}$ makes this method a good strategy for efficient insulation of micro- and nanoelectrodes contacting 2D semiconductor-based devices.
\end{abstract}




\section{WILEY-VCH}

\section{Introduction}

2D semiconducting materials, such as transition metal dichalcogenides (TMDCs ${ }^{[1]}$ and their heterostructures, are quickly emerging as the central component of a variety of nanoelectronic devices. ${ }^{[2-6]}$ One class of such devices can be used as (bio)sensors for electrical or electrochemical detection of (bio)molecules in a liquid. ${ }^{[7-14]}$ For this purpose, the 2D material is contacted by electrodes and exposed to a solution containing specific molecules that influence the electrical properties of the material. In solution, an electron transfer can occur between the electrode contacts and the interfering redox-active species. Therefore, it is crucial to passivate the electrodes and ensure that the signal originates solely from the sensing material. The most efficient way to eliminate such electron transfer is to coat the electrodes with a non-porous insulating barrier, which completely prevents the solution from coming into contact with the electrodes. ${ }^{[12,15,16]}$ This coating has to provide good insulating properties, strong adhesion to the electrode surface and stability under the device’s operating conditions. Furthermore, the method by which the coating is deposited needs to be compatible with the $2 \mathrm{D}$ material itself, as well as the microfabrication steps involved in device fabrication. This is especially important for devices that require the $2 \mathrm{D}$ material to remain free of contamination at the atomic level. One example of such a device is a field-effect transistor (FET) coupled with a nanometer-sized hole (nanopore-FET) for label-free single-molecule detection. ${ }^{[17-20]}$ In these devices, the 2D material is suspended over an aperture and should stay free of additional layers and contamination to allow the creation of a nanopore and to provide the best possible spatial resolution for sensing. ${ }^{[21]}$

Thin-films prepared by conventional deposition techniques, such as chemical vapor deposition (CVD), atomic layer deposition (ALD), sputtering, evaporation and other methods typically used in the microfabrication industry, are adapted for semiconducting devices operating in dry condition. Consequently, they suffer from various limitations, such as poor (electro)chemical stability that results in structural changes ${ }^{[22]}$ and dissolution ${ }^{[23,24]}$ (Figure S1a 


\section{WILEY-VCH}

and b), high porosity ${ }^{[25]}$, and delamination (Figure S1c) due to poor adhesion ${ }^{[26]}$. This results in an incomplete insulation rendering the devices inoperative. A special problem is the incompatibility of the different processing steps at the microscale: micro- and nanoscale patterning frequently require the usage of a photoresist mask, which leaves polymer residues on the coated 2D material. This can significantly affect the performance of the devices, and in some cases make them completely unusable. Furthermore, the mask can melt at the high temperatures used in some processes, for example, ALD deposition, limiting the pattern resolution (Figure S1d and e) and preventing the layer from depositing on the electrodes. ${ }^{\text {[27] }}$

In this work, we use a completely different approach in blocking the electrochemical reactions on the electrodes contacting a 2D TMDC semiconducting material, based on the electrochemical deposition of a polymer. ${ }^{[28,29]}$ By applying a voltage to the electrode in a solution containing certain polymer precursors (monomers), a variety of electrically nonconductive, polymer films of controllable thickness can be deposited on the electrode surface. This method is a simple and area selective deposition technique that works even at the nanoscale, where conventional photolithography processes fail. While being permeable to small molecules of gases and solvents, these polymer films are still compact and dense enough to efficiently prevent the access of electroactive species to the electrode surface. Since the polymer is non-conductive, the probability of an electron tunneling through the polymer barrier decreases exponentially with the distance between the electrode and the electroactive species. ${ }^{[30]}$ Therefore, even relatively thin films $(<100 \mathrm{~nm})$ can suppress the electron transfer by several orders of magnitude.

To ensure that the deposition does not take place on the semiconducting material, does not damage it or negatively affect its properties, it is crucial to choose the appropriate coating strategy, precursors, and experimental conditions. In this paper, we focus on molybdenum disulfide $\left(\mathrm{MoS}_{2}\right)$ as a semiconducting material, which is the most investigated TMDC semiconductor crystal. A monolayer of $\mathrm{MoS}_{2}$ (hexagonal $1 \mathrm{H}-\mathrm{MoS}_{2}$ phase) has a direct bandgap 


\section{WILEY-VCH}

of $1.9 \mathrm{eV}^{[31,32]}$, which enables a high current $\mathrm{ON} / \mathrm{OFF}$ ratio making it a promising candidate for field-effect transistors. ${ }^{[2,33]}$ Structural defects, such as sulfur vacancies, cause $\mathrm{MoS}_{2}$ to behave like an n-type semiconductor. ${ }^{[34,35]}$ The potential range in which the $\mathrm{MoS}_{2}$ crystal is electrochemically stable is between $-1.2 \mathrm{~V}$ and $1.3 \mathrm{~V}$ towards the $\mathrm{Ag} \mid \mathrm{AgCl}$ electrode. ${ }^{[36]}$ Since the rate of electron transfer between the n-type semiconductor and the species in solution is extremely slow at positive potentials ${ }^{[37-40]}$, this anodic window before the threshold potential for material oxidation could be utilized for the selective coating of electrodes contacting $\mathrm{MoS}_{2}$. One of the polymers which can be electrochemically deposited in this anodic region, and shows excellent insulating properties, is poly(phenylene oxide) (PPO). ${ }^{[41]}$ The deposition process is self-limiting, meaning that electropolymerization is terminated once the electrode surface is fully blocked, which results in reproducible coatings of less than $20 \mathrm{~nm}$ in thickness. ${ }^{[41-43]}$

Here, we investigate the applicability of PPO polymer electrodeposition as a method for the passivation of electrodes contacting $\mathrm{MoS}_{2}$ monolayer. To avoid possible oxidation and damage of the $\mathrm{MoS}_{2}$ monolayer at the contact with the electrodes, we perform the electrodeposition at mild conditions with a minimal number of cycles. Firstly, we analyze the deposited PPO thinfilm. The blocking properties of the PPO film were characterized electrochemically, the chemical composition was analyzed by X-ray photoelectron spectroscopy (XPS), and the surface morphology was inspected by scanning electron microscopy (SEM) and atomic force microscopy (AFM). Secondly, we investigate the compatibility of the method with electrodes in contact with high-quality single-crystal $\mathrm{MoS}_{2}$ layers. To demonstrate the applicability of this method at the nanoscale, we utilize it for coating the electrodes that are in contact with $\mathrm{MoS}_{2}$ nanoribbons of a nanopore - FET sensing device. We inspect the cleanliness of the 2D material at the atomic level using high-resolution transmission electron microscope (HR-TEM).

\section{Results and Discussion}




\section{WILEY-VCH}

\subsection{PPO Thin-Film Deposition}

The electrochemical deposition of the PPO layer and its properties were initially studied on macroscopic electrodes (platinum disc electrode (PtE) and a glassy carbon disc electrode (GCE) with $A=0.317 \mathrm{~cm}^{2}$ ), in order to obtain results with better statistical relevance (i.e. averaged over a larger area of electrode surface).

The schematic of the setup is shown in Figure 1a. PPO deposition was done by cyclic voltammetry in acetonitrile solution containing phenol and tetramethylammonium hydroxide. During the anodic oxidation of phenol, phenoxy radicals are generated, which then quickly polymerize into PPO. ${ }^{[28]}$ A diffusion path length, $l$, of the formed radicals can be calculated by $l=\sqrt{D \times \tau}$, where $D$ is the diffusion coefficient and $\tau$ is the half-life. Since $D$ of the phenol is on the order of $10^{-6} \mathrm{~cm}^{2} / \mathrm{s}{ }^{[44]}$, and $\tau$ of the produced monomer radicals is on the order of microseconds $^{[45]}$, their diffusion path length is also extremely short $(<100 \mathrm{~nm})$, which results in a thin reaction layer around the electrode making the deposition highly selective. Figure $1 \mathrm{~b}$ shows the corresponding cyclic voltammogram on PtE. In the first polarization cycle, an anodic wave appears in the range between 100 - $800 \mathrm{mV}$ vs. saturated calomel electrode (SCE), which corresponds to irreversible oxidation of phenol. The created PPO layer is electrically nonconductive $^{[28]}$ and quickly blocks the electrode surface, resulting in an anodic current decay. As previously reported ${ }^{[41]}$, the current is greatly suppressed after the first cycle. After the second cycle, the oxidation peak vanishes completely (Figure 1b, inset), which indicates the complete blockage of the electrode surface with non-conductive PPO.

Previous publications on PPO coating performed additionally steps to improve and stabilize the PPO film. In our case, these methods were not possible because of the damage they could do to the electrodes or to the underlying semiconducting material. For example, long-lasting cyclization in water up to relatively high voltages ${ }^{[43,46,47]}$ can result in oxidation ${ }^{[28,46]}$ and detachment of the electrodes (Figure S2a), as well as the semiconducting material ${ }^{[36]}$. Therefore, 


\section{WILEY-VCH}

we restricted the electrodeposition to a maximal voltage of $1.2 \mathrm{~V}$ vs. SCE up to which $\mathrm{MoS}_{2}$ is electrochemically stable. ${ }^{[36]}$ Moreover, we performed the deposition in acetonitrile, which additionally suppresses the oxidation of both the $\mathrm{MoS}_{2}{ }^{[48,49]}$ and the electrodes. To enhance the polymer cross-linking and improve the stability of the thin-films, some research-groups performed thermal annealing of the coatings at up to a few hundreds of degrees. ${ }^{[24,46]}$ However, we also avoid any kind of heat treatment since it can completely delaminate the coating at the micro- and nanoscale (Figure S2b and c).

\subsection{PPO Thin-Film Characterization}

The PPO film on PtE was characterized by $\mathrm{CV}$ in an aqueous solution of redox probes typically used in electrochemistry: ferrocyanide/ferricyanide $\left(\left[\mathrm{Fe}(\mathrm{CN})_{6}\right]^{4-/ 3-}\right)$ as an inner-sphere and hexaammineruthenium (III) $\left[\mathrm{Ru}\left(\mathrm{NH}_{3}\right)_{6}\right]^{3+}$, as an outer-sphere redox probe, respectively. The corresponding cyclic voltammograms after the coating were compared to the ones obtained on the bare PtE and are presented in Figure 1c and Figure S3a. After the coating, the cathodic and anodic peak current is decreased for at least one order of magnitude. The cyclic voltammograms became sigmoidal (Figure S3a and c), which can originate from two possible sources. One possibility is that the diffusion transport of the redox species through the PPO film is significantly slower than the diffusion through the solution. The second is the formation of pinholes, i.e. holes smaller than a few micrometers, in the polymer structure during the deposition. If the pinholes are separated from each other by a distance that is much larger than the pinhole diameter, the voltammetric current becomes controlled by radial mass transport, which results in a sigmoidal characteristic. ${ }^{[50]}$ Since we have not observed pinholes in our microscopy characterization (see below), the reason for the sigmoidal voltammograms of the coated electrodes must be the slower diffusion transport through the film. Similar behavior was also observed on the GCE (Figure S3b and c). 


\section{WILEY-VCH}

Voltage sweeps performed in $0.1 \mathrm{M} \mathrm{KNO}_{3}$ without the redox couple, show the blocking characteristics of the PPO film in the medium without redox-active species present (Figure 1d). The electrolytic decomposition of water, i.e. the processes of hydrogen and oxygen evolution, is pushed further toward the cathodic and anodic region, respectively, by approx. $300 \mathrm{mV}$ compared to bare Pt. In comparison with bare Pt, PPO coated Pt does not exhibit an anodic wave at potentials greater than $400 \mathrm{mV}$ that originates from the formation of surface Pt oxides. The potential window in which the coating is usable generally depends on the type of sensing device and its passivation requirements (we discuss this in more detail in the Electrode passivation requirements section). However, for most applications, the coating can be used within the potential window where the Faradaic current is almost completely blocked, approximately $2.3 \mathrm{~V}$ wide, and limited by electrolytic decomposition of water.

We tested, as well, the short-term stability of the PPO coating during the redox reaction of the $\left[\mathrm{Fe}(\mathrm{CN})_{6}\right]^{4-3-}$ (Figure S4). The experiments were performed under hydrodynamic and steady state conditions during $2 \mathrm{~h}$ and $22 \mathrm{~h}$, respectively. No change in PPO blocking properties was observed, which shows an excellent electrochemical stability and is in agreement with previous reports. ${ }^{[28,43]}$

The XPS results confirm that the Pt surface is almost completely covered with PPO (the spectra and more details can be found in Supplementary Information, Figure S5, Table S1 and S2). The fitting of the O 1s signal reveals that the PPO layer consists of two types of phenyl(Ph) based units: -O-Ph-O- and - $\mathrm{Ph}-\mathrm{OH}$ (Figure 2a), where the molar fraction of -Ph-OH units is approximately 20\%. The absence of $\mathrm{Cl}$ and $\mathrm{N}$ signal suggests that perchlorate and tetramethylammonium ions, respectively, did not incorporate into the polymer structure. This indicates that the polymer is uncharged. The electrodeposition of a charged polymer would result in simultaneous integration of the oppositely charged ions into the polymer. Since perchlorate and tetramethylammonium ions are the only ions that might get incorporated during 


\section{WILEY-VCH}

the deposition, the absence of both of their signals indicates that the PPO layer is neutrally charged.

The SEM micrographs (Figure S6a and b) of several $\mu \mathrm{m}^{2}$ area show a uniform mass contrast of PPO film deposited on Pt, which indicates that the film is homogeneous and of uniform thickness at this scale. This was additionally confirmed by SEM micrographs of the film deposited on the graphite surface (more details can be found in Supplementary Information and Figure S6c and d).

The AFM imaging was performed on a PPO film deposited on highly oriented pyrolytic graphite (HOPG) since the surface roughness of the underlying Pt substrates was relatively large compared to the roughness of the PPO film. As visible from Figure 2b and c, the nonuniformity of the PPO coating layer becomes more pronounced at the sub-micrometer scale. Additional micrographs of the PPO coating on Pt and HOPG substrates can be found in Figure S7 and S8, respectively.

\subsection{Compatibility with 2D $\mathrm{MoS}_{2}$}

In this part, we investigate the compatibility of the method with $\mathrm{MoS}_{2}$ semiconducting material. In all fabricated devices we use high-quality single-crystal mono- or bilayers grown by chemical vapor deposition (CVD). Ideally, the coating should not interfere or deposit on the $\mathrm{MoS}_{2}$ material. Since the PPO deposition occurs during the anodic process at highly positive potentials, we expect that $\mathrm{MoS}_{2}$, as the n-type semiconductor, should stay inactive in these conditions.

\subsubsection{Supported $\mathrm{MoS}_{2}$ devices}

The schematic of the set-up is shown in Figure 3a. The device consists of several pairs of electrodes (Figure 3b) where each pair is contacting one single-crystal $\mathrm{MoS}_{2}$ layer (Figure 3c). The gap between the electrodes was designed to be significantly larger than the diffusion path length of the phenoxy radicals to prevent them to diffuse and polymerize at the other 


\section{WILEY-VCH}

electrode. Figure 3d shows an AFM image of one of the contacted crystals before the coating of the electrode. Connecting the left electrode as the working electrode during coating results in the deposition of the PPO layer. This is immediately visible from the AFM images, which show an increase in the height profile (Figure 3e) of $11.3 \pm 0.9 \mathrm{~nm}$ consistent with the thickness obtained in previous reports. ${ }^{[43]}$ A 3D AFM micrograph of the same area is shown in Figure 3f. The cross-sections taken before and after the electrode coating reveal that the height of the $\mathrm{MoS}_{2}$ did not change (Figure 3e and Figure S9), indicating that there is no PPO deposition on $\mathrm{MoS}_{2}$.

To confirm that the contact between the $\mathrm{MoS}_{2}$ and the electrode was not oxidized or damaged during the deposition of PPO on the Pt electrode overlaying $\mathrm{MoS}_{2}$, we measure I-V characteristics in a two-terminal configuration in dry conditions before and after the coating (Table S3 and Figure S10). Before the coating, the conductance of the $\mathrm{MoS}_{2}$ is on the order of a few pS, since the conductance in thin $\mathrm{MoS}_{2}$ (less than 5 layers) is highly dominated by the contact resistance. ${ }^{[51-53]}$ To confirm that the devices are operational, the devices were annealed in-situ in vacuum. This significantly reduced the Schottky barrier and contact resistance ${ }^{[51,54]}$, resulting in a dramatic rise of the conductance of approximately four orders of magnitude. After coating the electrodes, the $\mathrm{MoS}_{2}$ conductance was measured again. The coated devices, as well as the control group (uncoated devices exposed to the same conditions), showed largely the same conductance (Table S3). The values are comparable to the initial values obtained in air before the coating, which confirmed that the contacts remained intact and the $\mathrm{MoS}_{2}$ crystal was still conductive.

\subsubsection{Suspended $\mathrm{MoS}_{2}$ devices}

After confirming that the contacts to the $\mathrm{MoS}_{2}$ are unaffected by the coating method, we set out to test the applicability of our method to a $\mathrm{MoS}_{2}$ based biosensing device designed for single-molecule detection. Figure 4a and b show the schematic representation and an optical 


\section{WILEY-VCH}

micrograph of the device, respectively. Three nanoribbons of $\mathrm{MoS}_{2}$ and corresponding metal contacts are fabricated on top of a $20 \mathrm{~nm}$ thick $\mathrm{SiN}_{\mathrm{x}}$ membrane. The ribbon located in the middle of the device is placed on top of an $80 \mathrm{~nm}$ large aperture, making it effectively freestanding. A nanopore can then be drilled into this freestanding part to deliver molecules to the sensor. ${ }^{[20]}$

The thin $\mathrm{SiN}_{\mathrm{x}}$ membrane, as well as the freestanding $\mathrm{MoS}_{2}$, allow the inspection of the coating method at the atomic scale by using the high-resolution transmission electron microscopy (HR-TEM) technique. To reduce the chances of damage and contamination of the 2D material during imaging, we performed the imaging at a reduced acceleration voltage of 80 $\mathrm{kV} \cdot{ }^{[55,56]}$ We inspected the $\mathrm{MoS}_{2}$ ribbons after the coating process was completed. One of the ribbons was used as a control, keeping the contacting electrodes uncoated. On both, the out-offocus (Figure 4c) and the focused close-up (Figure 4d) images, the $\mathrm{MoS}_{2}$ ribbons connected to the coated electrodes show similar contrast as the control ribbon. This confirms that the PPO deposition did not occur on $\mathrm{MoS}_{2}$ during the electrode coating, which is consistent with the AFM results (Figure 3e and Figure S9). The inspection of the freestanding $\mathrm{MoS}_{2}$ region reveals atomically clean parts after the electrode coating (Figure 4e and f). The contamination visible on the suspended part is most likely not caused by the electrochemical deposition, primarily because of the inhomogeneous distribution. Typically this contamination is associated with poly(methyl methacrylate) (PMMA) residues used in the device fabrication. ${ }^{[14]}$ Another possibility is the contamination caused by the interaction of the electron beam with the resist residues, PPO coating, or residual phenylene oxide oligomers that were not completely washed away. However, the existence of clean regions after the coating process (Figure 4f) further supports the observation that electrochemical deposition does not take place on $\mathrm{MoS}_{2}$. There are two main reasons why the PPO deposition does not occur on the $\mathrm{MoS}_{2}$ : the semiconducting nature of $\mathrm{MoS}_{2}$ (in this case n-type semiconductor), and the non-efficient charge injection into the 2D material due to the high contact resistance at the electrode - 2D material interface. It has 


\section{WILEY-VCH}

been observed that in n-type $\mathrm{MoS}_{2}$ the heterogeneous electron transfer (HET) rate decreases with more positive potentials. ${ }^{[39,40]}$ For redox couples that have relatively positive redox potentials, such as $\mathrm{Fe}^{3+/ 2+}$, the HET readily occurs only in the cathodic direction (reduction), while the anodic reaction (oxidation) gets partially or completely blocked. ${ }^{[37]}$ The reason for this blockage is that during the anodic polarization of the $\mathrm{MoS}_{2}$ the number of available charge carriers in the material (electrons), is reduced, which results in lower exchange currents. High contact resistance, which is a general problem in device fabrication, also plays a role by limiting the charge carrier injection and subsequently the electron transport through the 2D material. ${ }^{[39,57]}$ This might also be the reason why we have not observed any deposition at the sites which could potentially become electrochemically active, particularly, the edges and defective sites where molybdenum atoms are exposed to the solution. ${ }^{[37,38,40,58]}$ However, lowering the contact resistance by locally transforming $1 \mathrm{H}$ phase to the metallic $1 \mathrm{~T}$ phase $\mathrm{e}^{[59]}$, or increasing the density of defects ${ }^{[60]}$, might in some cases enhance the activity of the basal plane $^{[61,62]}$ and affect the average HET rate.

\subsection{Electrode passivation requirements}

In contrast to conventional electrochemical measurements with a three-electrode system, in devices with two electrode contacts, the anodic (oxidation) and cathodic (reduction) processes are highly dependent on each other. The measured current will be limited by the electrode with the electrochemical process that results in the lowest exchange current, which depends on the electron transfer rate and electrode area exposed to the solution. Therefore, when designing a sensor, an important parameter is the exposed area of the contact electrodes. Reducing the exposed area also reduces the amount of "leakage", i. e. current through the solution exchanged between the two electrodes. By defining an upper limit for the acceptable fraction of the current leakage, $x$, compared to the signal measured, we can estimate the maximal electrode area, $A$, allowed to be exposed to the solution: $=x \cdot V \cdot G_{M o S 2} /[j \cdot(1-x)]$ 


\section{WILEY-VCH}

, where $G_{\text {Mos2 }}$ is the $\mathrm{MoS}_{2}$ conductance and $j$ is the current leakage density at the voltage $V$. To estimate the current leakage density between two PPO coated electrodes in the typical biosensing operating conditions, we performed an $I-V$ scan in a two-electrode configuration in a presence the redox-active probe, $\left[\mathrm{Fe}(\mathrm{CN})_{6}\right]^{4-3-}$. To ensure that the measured current originates exclusively from the leakage through the solution, we used a device without $\mathrm{MoS}_{2}$ as a control. With the PPO coating, the current is almost completely blocked (Figure 5) and we get a current leakage density of approximately $20 \mu \mathrm{A} / \mathrm{cm}^{2}$ at $500 \mathrm{mV}$. Calculating with a leakage fraction of $10 \%$ and a very conservative $\mathrm{MoS}_{2}$ conductance value of $100 \mathrm{pS}$, for a device that operates up to $500 \mathrm{mV}$, an area of $25 \mu \mathrm{m}^{2}$ on each electrode could be exposed to the solution. Since the true signal depends on the conductance of $\mathrm{MoS}_{2}$ material, the current leakage fraction can also be tuned by adjusting the geometry and the aspect ratio of the nanoribbon, and by minimizing the contact resistance ${ }^{[57]}$.

\section{Conclusion}

The passivation of electrode contacts is crucial for the performance of electrical and electrochemical sensing devices operating in liquid. The main reason is that the Faradaic current resulting from the electron transfer between the electrodes and the redox-active species can mask the true signal of the sensing material.

We have shown that the electrochemical deposition of the PPO polymer is suitable for passivating electrodes in contact with a $\mathrm{MoS}_{2} 2 \mathrm{D}$ semiconductor. The electropolymerization of the PPO polymer results in the deposition of a thin-film with an almost complete electron transfer blockage in the potential window of approximately $2.3 \mathrm{~V}$. The deposition process occurs at mild conditions, with a minimal number of cycles, and in the potential window where $\mathrm{MoS}_{2}$ is inert. This results in a highly selective deposition, controllable and compatible with $\mathrm{MoS}_{2}$ and the electrode material. 


\section{WILEY-VCH}

Our method avoids the problems that typical deposition techniques can suffer from, including the contamination with photoresist mask residues, which can significantly affect the performance of the devices. In our approach, a mask is not needed: each electrode can be individually addressed and coated separately by connecting it as the working electrode.

The recent progress on wafer-scale CVD growth of high-quality 2D semiconducting materials and their heterostructures is going to enable large-scale production of (bio)sensing devices of various architectures. Further miniaturization of electrodes and sensing material to the nanoscale will enable (bio)sensors-on-chip integration which will bring high-throughput, and therefore, more accurate detection of different analytes. This requires fabrication processes reliable at the nanoscale and compatible with 2D semiconductors. To the best of our knowledge, this is the first report on a highly selective electrodeposition method for coating the electrodes that are in contact with 2D TMDC semiconductors, like $\mathrm{MoS}_{2}$.

This approach can be further extended to other types of polymers and electrodes, as well as other 2D semiconducting materials. In principle, any polymer with satisfying current blocking properties and that electropolymerizes in the potential window where the semiconductor is inactive is a good candidate for the application. Analogously, any semiconducting material that is electrochemically inactive in the conditions in which the electrodeposition occurs, is compatible with this method. This opens a broad spectrum of different combinations of polymers and 2D semiconducting materials. Furthermore, since the HET rate in 2D semiconductors can be nicely regulated by applying different back gate potentials ${ }^{[63,64]}$, the FET devices with incorporated back gate could use this advantage to keep the 2D semiconductor in the OFF state during the coating of the electrode contacts. We believe that our demonstration of the method on the devices build on single-crystal $\mathrm{MoS}_{2}$ monolayers will be useful for future (bio)sensing devices, especially FET, which are based on 2D TMDCs for label-free detection and rely on an electronic readout. The highly selective insulation, the simple approach, and the 


\section{WILEY-VCH}

compatibility with $\mathrm{MoS}_{2}$ and potentially with other TMDCs, makes this method a good strategy for efficient insulation of micro- and nanoelectrodes of 2D semiconductor-based devices.

\section{Experimental Section}

All the reagents and solvents used were analytical grade and purchased from Sigma - Aldrich, Merck, Darmstadt, Germany. PtE and GCE electrodes: Platinum disc electrode (PtE) (d = 0.635 cm, BASinc, West Lafayette, USA) and glassy carbon disc electrode (GCE) $(\mathrm{d}=0.635 \mathrm{~cm}$, BASinc, West Lafayette, USA) were used. After polishing with $0.3 \mu \mathrm{m} \mathrm{Al}_{2} \mathrm{O}_{3}$, the electrodes were rinsed and sonicated in acetone, isopropanol, and deionized water. PtE was activated by cyclic polarization from $1550 \mathrm{mV}$ to $-300 \mathrm{mV}$ vs. saturated calomel electrode (SCE) $\mathrm{Hg}$ | $\mathrm{Hg}_{2} \mathrm{Cl}_{2} \mid \mathrm{KCl}(3.5 \mathrm{M})$ (BASinc, West Lafayette, USA) in $0.1 \mathrm{M}$ perchloric acid at a scan rate of $100 \mathrm{mV} / \mathrm{s}$.

Substrates for XPS, SEM and AFM characterization: Pt (60 nm in thickness) with $5 \mathrm{~nm} \mathrm{Ti}$ adhesion layer was evaporated onto silicon substrates (360 $\mu \mathrm{m}$ silicon Si / $60 \mathrm{~nm}$ silicon dioxide $\left(\mathrm{SiO}_{2}\right) / 20 \mathrm{~nm}$ silicon nitride $\left(\mathrm{SiN}_{\mathrm{x}}\right)$ ). Graphite and Highly Oriented Pyrolytic Graphite (HOPG ZYH/1mm, MikroMasch, Watsonville, USA) crystals were micromechanically exfoliated by the scotch tape-based method and transferred onto Pt evaporated on a silicon substrate.

$\mathrm{MoS}_{2}$ transfer: $\mathrm{MoS}_{2} 2 \mathrm{D}$ monocrystals were obtained by CVD growth on sapphire and characterized as previously reported. ${ }^{[65]}$ The thickness was additionally confirmed by optical contrast and AFM measurements. Monocrystals were transferred onto the target substrate by our custom wet transfer method described elsewhere. ${ }^{[14]}$

Supported $\mathrm{MoS}_{2}$ devices: All fabrication steps were done on a $360 \mu \mathrm{m}$ silicon wafer containing $60 \mathrm{~nm} \mathrm{SiO} 2$ and $20 \mathrm{~nm} \mathrm{SiN}$ x layers. Electrodes were patterned on the $\mathrm{SiN}_{\mathrm{x}}$ by a combination 


\section{WILEY-VCH}

of photolithography (large dimensions) and e-beam lithography (EBL, small dimensions) and metal evaporation. Low contact resistance between the electrode and the $\mathrm{MoS}_{2}$ has been shown to be crucial for the performance of devices and, as such, has been widely studied. ${ }^{[57]}$ In general, it is challenging to achieve a low resistance contact on $1 \mathrm{H}-\mathrm{MoS}_{2}$ phase. For contacting $\mathrm{MoS}_{2}$ we use $70 \mathrm{~nm}$ gold $(\mathrm{Au})$, the most commonly used ${ }^{[59,66,67]}$ material for that purpose, in combination with $5 \mathrm{~nm}$ titanium (Ti) as an adhesion layer. Ti is a metal with a low workfunction, which can covalently bind to $\mathrm{MoS}_{2}$ at the interface to reduce the contact resistance ${ }^{[68-}$ ${ }^{70]}$. On the top of the Au we evaporate an additional layer of $5 \mathrm{~nm}$ Pt. Prior to PPO deposition, the devices were rinsed with acetone and isopropanol, and carefully blown dried with nitrogen. Each device was fixed to a custom made PCB board, the electrodes were wire-bonded to the PCB contacts, which were then connected as a working electrode in a three-electrode setup.

Suspended $\mathrm{MoS}_{2}$ devices: Prior to $\mathrm{MoS}_{2}$ transfer, we created $30 \mu \mathrm{m}$ x $30 \mu \mathrm{m} \mathrm{SiN}_{\mathrm{x}}$ membrane with an aperture (80 $\mathrm{nm}$ in diameter) by using electron beam lithography (EBL), reactive ion etching (RIE) and wet etching in a hot potassium hydroxide solution (30\% w/w). The protocol for this process is available elsewhere. ${ }^{[14]}$ After the $\mathrm{MoS}_{2}$ transfer and cleaning, EBL was used to pattern electrodes ( $5 \mathrm{~nm} \mathrm{Ti} / 70 \mathrm{~nm} \mathrm{Au} / 5 \mathrm{~nm} \mathrm{Pt}$ ) on top of the $\mathrm{MoS}_{2}$. After patterning these contacts, the $\mathrm{MoS}_{2}$ monolayer is etched into nanoribbons ( $2 \mu \mathrm{m}$ x $500 \mathrm{~nm}$ ) using EBL and RIE ( $\mathrm{O}_{2}$ plasma). In all EBL steps a 3-step alignment scheme was used to achieve the necessary precision between individual lithographic steps. Prior to PPO deposition, the devices were rinsed with acetone and isopropanol, and carefully blown dried with nitrogen. Each device was fixed to a PCB board, the electrodes were wire-bonded to the PCB contacts, which were then connected as a working electrode in a three-electrode setup. The number of tested devices is indicated in the section Number of Devices in the Supplementary Information.

PPO deposition: PPO deposition was done by cyclic voltammetry (CV) in a solution of phenol (50 mM), tetramethylammonium hydroxide pentahydrate, $\mathrm{TMAH}^{*} 5 \mathrm{H}_{2} \mathrm{O}(50 \mathrm{mM})$, and 


\section{WILEY-VCH}

tetrabutylammonium perchlorate, TBAP $(100 \mathrm{mM})$ in acetonitrile. The working electrode was polarized during 10 cycles from $-200 \mathrm{mV}$ to $1250 \mathrm{mV}$ vs. SCE at $50 \mathrm{mV} \mathrm{s}^{-1}$. In all measurements, the counter electrode was a platinum wire (Sigma-Aldrich, Merck, Darmstadt, Germany) while SCE combined with a salt bridge was used as a reference. The measurements were acquired with PalmSens2 (Palm Instruments BV, Houten, Netherlands) potentiostat. After deposition, the electrodes and the devices were thoroughly rinsed with acetonitrile to remove the adsorbed phenol and TMAH, and gently dried with a nitrogen flow.

Electrochemical characterization on PtE and GCE: Cyclic voltammograms were recorded in $0.1 \mathrm{M} \mathrm{KNO}_{3}$ water solution with and without the redox-active probe. Redox-active probes used were: hexaammineruthenium (III) chloride, $\left[\mathrm{Ru}\left(\mathrm{NH}_{3}\right)_{6}\right] \mathrm{Cl}_{3}(1 \mathrm{mM})$, and equimolar solution potassium ferrocyanide/ferricyanide, $\mathrm{K}_{4}\left[\mathrm{Fe}(\mathrm{CN})_{6}\right] / \mathrm{K}_{3}\left[\mathrm{Fe}(\mathrm{CN})_{6}\right](1 \mathrm{mM}$ and $5 \mathrm{mM})$ respectively. The scan rate was $50 \mathrm{mV} \mathrm{s}^{-1}$.

X-Ray Photoelectron Spectroscopy (XPS). Measurements were carried out using a PHI VersaProbe II scanning XPS microprobe (Physical Instruments AG, Germany). The analysis was performed using a monochromatic $\mathrm{Al} \mathrm{K \alpha} \mathrm{X}$-ray source of $24.8 \mathrm{~W}$ power with a beam size of $100 \mu \mathrm{m}$. The spherical capacitor analyzer was set at $45^{\circ}$ take-off angle with respect to the sample surface. The pass energy was $46.95 \mathrm{eV}$ yielding a full width at half maximum of 0.91 $\mathrm{eV}$ for the Ag 3d 5/2 peak. Curve fitting was performed using the XPSPEAK 4.1 software (https://xpspeak.software.informer.com/4.1/). The corresponding chemical structure was drawn in MolView, an open-source web-application (http://molview.org/).

Scanning Electron Microscopy: All SEM images were acquired using an ultra-high resolution field emission gun SEM, Zeiss Merlin (Carl Zeiss Microscopy GmbH, Germany), at acceleration voltages of either $500 \mathrm{~V}, 1 \mathrm{kV}$, and $3 \mathrm{kV}$. 


\section{WILEY-VCH}

Atomic Force Microscopy: The devices and substrates were imaged using microcantilevers (70

$\mathrm{kHz}, 2 \mathrm{~N} \mathrm{~m}^{-1}$, Olympus) and an AFM Asylum Cypher (Oxford Instruments - Asylum Research, Santa Barbara, USA) operating in tapping (AC) mode.

Transmission Electron Microscopy: The devices were imaged by a transmission electron microscope (FEI Talos, Hillsboro, Oregon, USA) at an acceleration voltage of $80 \mathrm{kV}$.

Current-voltage measurements in dry conditions: The conductance measurements of contacted $\mathrm{MoS}_{2}$ monocrystals were performed in a two-terminal configuration in air at RT. The measurements were repeated in vacuum (base pressure $10^{-6} \mathrm{mbar}$ ) at RT after the annealing treatment of devices. Annealing was done at $140^{\circ} \mathrm{C}$ in vacuum for $24 \mathrm{~h}$. After PPO deposition on the electrode contacts, the conductance was measured again in air and at RT. Annealing was not performed to avoid damaging the coating as mentioned previously (Figure S2b and c)

\section{Supporting Information}

Supporting Information is available from the Wiley Online Library or from the author.

\section{Acknowledgements}

We thank Dr. Pierre Mettraux and Molecular and Hybrid Materials Characterization Center (MHMC) for the XPS measurement. We thank Prof. Carlotta Guiducci for a valuable discussion and the access to Potentiostat. Also, we would like to thank Prof. Andras Kis for the access to Atomic Force Microscope and Probe station, Dumitru Dumcenco and Yanfei Zhao for providing $\mathrm{MoS}_{2}$ material, and the Centre Interdisciplinaire de Microscopie Electronique (CIME) at the École Polytechnique fédérale de Lausanne (EPFL) for access to electron microscopes. Furthermore, we thank Prof. Christophe Roussel, Dr. Ahmet Avsar and Alberto 


\section{WILEY-VCH}

Chiarrocchi for many helpful discussions. Device fabrication was partially carried out at the

EPFL Center for Micro/Nanotechnology (CMi). This work was financially supported by the Swiss National Science Foundation (SNSF) consolidator grant (BIONIC BSCGI0_157802), CCMX project (“Large-Area Growth of 2D Materials for device integration”), and EPFL's ENABLE program.

Received: ((will be filled in by the editorial staff))

Revised: ((will be filled in by the editorial staff)) Published online: ((will be filled in by the editorial staff))

\section{References}

[1] S. Manzeli, D. Ovchinnikov, D. Pasquier, O. V. Yazyev, A. Kis, Nat. Rev. Mater. 2017, 2, 17033.

[2] B. Radisavljevic, A. Radenovic, J. Brivio, V. Giacometti, A. Kis, Nat. Nanotechnol. 2011, 6, 147.

[3] O. Lopez-Sanchez, D. Lembke, M. Kayci, A. Radenovic, A. Kis, Nat. Nanotechnol. 2013, 8, 497.

[4] D. Unuchek, A. Ciarrocchi, A. Avsar, K. Watanabe, T. Taniguchi, A. Kis, Nature 2018, 560, 340 .

[5] J. Feng, M. Graf, K. Liu, D. Ovchinnikov, D. Dumcenco, M. Heiranian, V. Nandigana, N. R. Aluru, A. Kis, A. Radenovic, Nature 2016, 536, 197.

[6] D. Jariwala, V. K. Sangwan, L. J. Lauhon, T. J. Marks, M. C. Hersam, ACS Nano 2014, 8, 1102.

[7] L. Wang, Y. Wang, J. I. Wong, T. Palacios, J. Kong, H. Y. Yang, Small 2014, 10, 1101. 


\section{WILEY-VCH}

[8] D. Sarkar, W. Liu, X. Xie, A. C. Anselmo, S. Mitragotri, K. Banerjee, ACS Nano 2014, 8, 3992.

[9] J. Lee, P. Dak, Y. Lee, H. Park, W. Choi, M. A. Alam, S. Kim, Sci. Rep. 2014, 4, 1.

[10] K. Liu, J. Feng, A. Kis, A. Radenovic, ACS Nano 2014, 8, 2504.

[11] J. Feng, K. Liu, R. D. Bulushev, S. Khlybov, D. Dumcenco, A. Kis, A. Radenovic, Nat. Nanotechnol. 2015, 10, 1070.

[12] D.-W. Lee, J. Lee, I. Y. Sohn, B.-Y. Kim, Y. M. Son, H. Bark, J. Jung, M. Choi, T. H. Kim, C. Lee, N.-E. Lee, Nano Res. 2015, 8, 2340.

[13] S. Barua, H. S. Dutta, S. Gogoi, R. Devi, R. Khan, ACS Appl. Nano Mater. 2017, acsanm.7b00157.

[14] M. Graf, M. Lihter, M. Thakur, V. Georgiou, J. Topolancik, B. R. Ilic, K. Liu, J. Feng, Y. Astier, A. Radenovic, Nat. Protoc. 2019, 14, 1130.

[15] H. Nam, B. R. Oh, P. Chen, J. S. Yoon, S. Wi, M. Chen, K. Kurabayashi, X. Liang, Appl. Phys. Lett. 2015, 107.

[16] M. M. N. Nuzaihan, U. Hashim, M. K. Md Arshad, S. R. Kasjoo, S. F. A. Rahman, A. R. Ruslinda, M. F. M. Fathil, R. Adzhri, M. M. Shahimin, Biosens. Bioelectron. 2016, 83, 106.

[17] F. Traversi, C. Raillon, S. M. Benameur, K. Liu, S. Khlybov, M. Tosun, D. Krasnozhon, A. Kis, A. Radenovic, Nat. Nanotechnol. 2013, 8, 939.

[18] W. M. Parkin, M. Drndić, ACS Sensors 2018, 3, 313.

[19] S. J. Heerema, L. Vicarelli, S. Pud, R. N. Schouten, H. W. Zandbergen, C. Dekker, ACS Nano 2018, 12, 2623.

[20] M. Graf, K. Liu, A. Sarathy, J.-P. Leburton, A. Radenovic, Biophys. J. 2018, 114, 180a.

[21] G. Danda, M. Drndić, Curr. Opin. Biotechnol. 2019, 55, 124.

[22] L. H. Kim, K. Kim, S. Park, Y. J. Jeong, H. Kim, D. S. Chung, S. H. Kim, C. E. Park, 


\section{WILEY-VCH}

ACS Appl. Mater. Interfaces 2014, 6, 6731.

[23] A. I. Abdulagatov, Y. Yan, J. R. Cooper, Y. Zhang, Z. M. Gibbs, A. S. Cavanagh, R.

G. Yang, Y. C. Lee, S. M. George, ACS Appl. Mater. Interfaces 2011, 3, 4593.

[24] G. C. Correa, B. Bao, N. C. Strandwitz, ACS Appl. Mater. Interfaces 2015, 7, 14816.

[25] C. C. Yang, W. C. Chen, J. Mater. Chem. 2002, 12, 1138.

[26] V. K. Khanna, J. Phys. D. Appl. Phys. 2011, 44.

[27] R. H. J. Vervuurt, A. Sharma, Y. Jiao, W. M. M. Kessels, A. A. Bol, Nanotechnology 2016, 27.

[28] F. Bruno, M. C. Pham, Electrochim. Acta 1977, 22.

[29] T. Tevi, H. Yaghoubi, J. Wang, A. Takshi, J. Power Sources 2013, 241, 589.

[30] J. C. Fisher, I. Giaever, J. Appl. Phys. 1961, 32, 172.

[31] K. F. Mak, C. Lee, J. Hone, J. Shan, T. F. Heinz, Phys. Rev. Lett. 2010, 105, 136805.

[32] J. K. Ellis, M. J. Lucero, G. E. Scuseria, Appl. Phys. Lett. 2011, 99, 261908.

[33] M. Chhowalla, D. Jena, H. Zhang, Nat. Rev. 2016, 1, 16052.

[34] L. P. Feng, J. Su, Z. T. Liu, J. Alloys Compd. 2014, 613, 122.

[35] J. Su, N. Li, Y. Zhang, L. Feng, Z. Liu, AIP Adv. 2015, 5.

[36] X. Chia, A. Ambrosi, D. Sedmidubský, Z. Sofer, M. Pumera, Chem. - A Eur. J. 2014, 20, 17426.

[37] S. M. Ahmed, H. Gerischer, Electrochim. Acta 1979, 24, 705.

[38] S. M. Ahmed, Electrochim. Acta 1982, 27, 707.

[39] O. Henrotte, T. Bottein, H. Casademont, K. Jaouen, T. Bourgeteau, S. Campidelli, V. Derycke, B. Jousselme, R. Cornut, ChemPhysChem 2017, 18, 2777.

[40] N. L. Ritzert, V. A. Szalai, T. P. Moffat, Langmuir 2018, 34, 13864.

[41] R. L. Mccarley, R. E. Thomas, E. A. Irene, R. W. Murray, Film 1990, 290, 79.

[42] R. L. McCarley, E. A. Irene, R. W. Murray, J. Phys. Chem. 1991, 95, 2492.

[43] S. Deheryan, D. J. Cott, R. Muller, M. Timmermans, M. Heyns, P. M. Vereecken, 


\section{WILEY-VCH}

Carbon N. Y. 2015, 88, 42.

[44] R. Niesner, A. Heintz, J. Chem. Eng. Data 2000, 45, 1121.

[45] T. N. Das, J. Phys. Org. Chem. 2009, 22, 872.

[46] M. Y. Timmermans, F. Mattelaer, S. Moitzheim, N. Clerckx, A. Sepulveda, S. Deheryan, C. Detavernier, P. M. Vereecken, J. Appl. Polym. Sci. 2017, 134, n/a.

[47] A. Lipatov, B. B. Wymore, A. Fursina, T. H. Vo, A. Sinitskii, J. G. Redepenning, Chem. Mater. 2015, 27, 157.

[48] W. Kautek, H. Gerischer, Berichte der Bunsengesellschaft für Phys. Chemie 1980, 84, 645.

[49] W. Kautek, H. Gerischer, Electrochim. Acta 1982, 27, 1035.

[50] T. J. Davies, S. Ward-Jones, C. E. Banks, J. Del Campo, R. Mas, F. X. Muñoz, R. G. Compton, J. Electroanal. Chem. 2005, 585, 51.

[51] B. W. H. Baugher, H. O. H. Churchill, Y. Yang, P. Jarillo-Herrero, Nano Lett. 2013, $13,4212$.

[52] S. L. Li, K. Komatsu, S. Nakaharai, Y. F. Lin, M. Yamamoto, X. Duan, K. Tsukagoshi, ACS Nano 2014, 8, 12836.

[53] Y. Guo, Y. Han, J. Li, A. Xiang, X. Wei, S. Gao, Q. Chen, ACS Nano 2014, 8, 7771.

[54] A. T. S. Wee, Q. Wang, Y. Zheng, C. Han, W. Chen, D. Qi, J. Jiang, W. Zhang, 2D Mater. 2017, 4, 045016.

[55] H. P. Komsa, J. Kotakoski, S. Kurasch, O. Lehtinen, U. Kaiser, A. V. Krasheninnikov, Phys. Rev. Lett. 2012, 109, 1.

[56] H. J. Park, G. H. Ryu, Z. Lee, Appl. Microsc. 2015, 45, 107.

[57] A. Allain, J. Kang, K. Banerjee, A. Kis, Nat. Mater. 2015, 14, 1195.

[58] T. F. Jaramillo, K. P. Jørgensen, J. Bonde, J. H. Nielsen, S. Horch, I. Chorkendorff, Science (80-. ). 2007, 317, 100.

[59] R. Kappera, D. Voiry, S. E. Yalcin, W. Jen, M. Acerce, S. Torrel, B. Branch, S. Lei, W. 


\section{WILEY-VCH}

Chen, S. Najmaei, J. Lou, P. M. Ajayan, G. Gupta, A. D. Mohite, M. Chhowalla, APL Mater. 2014, 2.

[60] J. Su, N. Li, Y. Zhang, L. Feng, Z. Liu, AIP Adv. 2015, 5.

[61] D. Voiry, R. Fullon, J. Yang, C. de Carvalho Castro e Silva, R. Kappera, I. Bozkurt, D. Kaplan, M. J. Lagos, P. E. Batson, G. Gupta, A. D. Mohite, L. Dong, D. Er, V. B. Shenoy, T. Asefa, M. Chhowalla, Nat. Mater. 2016, 15, 1003.

[62] H. Li, C. Tsai, A. L. Koh, L. Cai, A. W. Contryman, A. H. Fragapane, J. Zhao, H. S. Han, H. C. Manoharan, F. Abild-Pedersen, J. K. Nørskov, X. Zheng, Nat. Mater. 2016, $15,48$.

[63] Y. Wang, C. H. Kim, Y. Yoo, J. E. Johns, C. D. Frisbie, Nano Lett. 2017, 17, 7586.

[64] Y. Wang, S. Udyavara, M. Neurock, C. Daniel Frisbie, Nano Lett. 2019.

[65] D. Dumcenco, D. Ovchinnikov, K. Marinov, P. Lazić, M. Gibertini, N. Marzari, O. L. Sanchez, Y.-C. Kung, D. Krasnozhon, M.-W. Chen, S. Bertolazzi, P. Gillet, A. Fontcuberta i Morral, A. Radenovic, A. Kis, ACS Nano 2015, 9, 4611.

[66] M. Li, N. Liu, P. Li, J. Shi, G. Li, N. Xi, Y. Wang, L. Liu, ACS Appl. Mater. Interfaces 2017, 9, 8361.

[67] N. Huo, J. Kang, Z. Wei, S. S. Li, J. Li, S. H. Wei, Adv. Funct. Mater. 2014, 24, 7025.

[68] J. Kang, D. Sarkar, W. Liu, D. Jena, K. Banerjee, Tech. Dig. - Int. Electron Devices Meet. IEDM 2012, 17.4.1.

[69] I. Popov, G. Seifert, D. Tománek, Phys. Rev. Lett. 2012, 108, 1.

[70] W. Liu, J. Kang, W. Cao, D. Sarkar, Y. Khatami, D. Jena, K. Banerjee, Tech. Dig. - Int. Electron Devices Meet. IEDM 2013, 19.4.1. 


\section{WILEY-VCH}
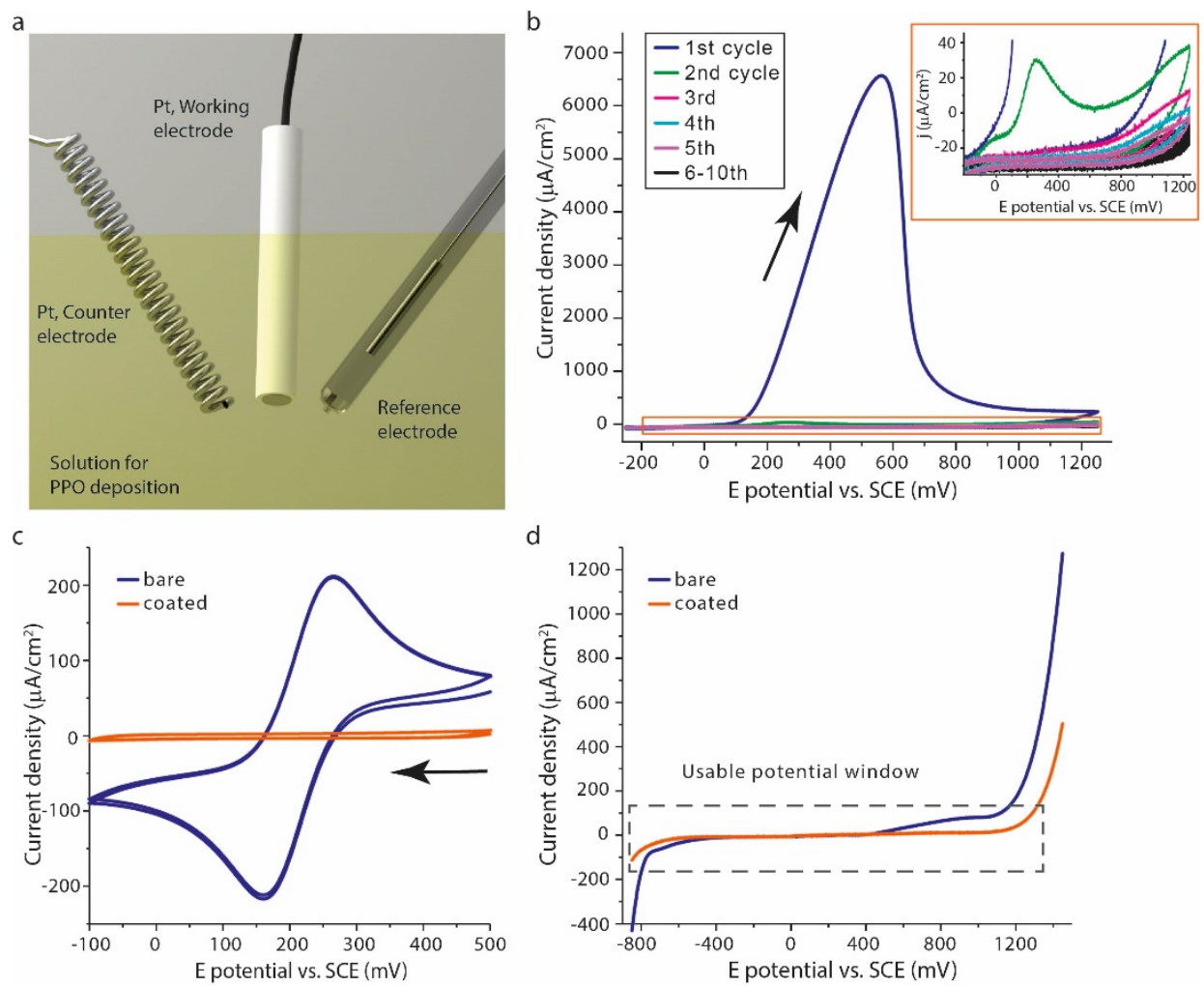

Figure 1. (a) Schematic of the three-electrode setup used for PPO deposition. The objects are not to scale. (b) Cyclic voltammogram of the PPO deposition on a Pt disc electrode (PtE). The arrow indicates the direction of the voltage sweep. The inset shows an enlarged voltammogram of the 2-10 deposition cycle. The deposition was performed in a solution of $50 \mathrm{mM}$ phenol, 50 mM TMAH*5H $\mathrm{H}_{2} \mathrm{O}$ and $0.1 \mathrm{M}$ TBAP in acetonitrile. (c) Cyclic voltammogram of PtE recorded in $1 \mathrm{mM}\left[\mathrm{Fe}(\mathrm{CN})_{6}\right]^{4-/ 3-}$ and $0.1 \mathrm{M} \mathrm{KNO}_{3}$ in water before and after PPO deposition. (d) Voltammogram of PtE recorded in $0.1 \mathrm{M} \mathrm{KNO}_{3}$. The scan was done starting from $0 \mathrm{~V}$ toward the cathodic and anodic region. The scan rate was $50 \mathrm{mV} \mathrm{s}^{-1}$ in all measurements. 


\section{WILEY-VCH}
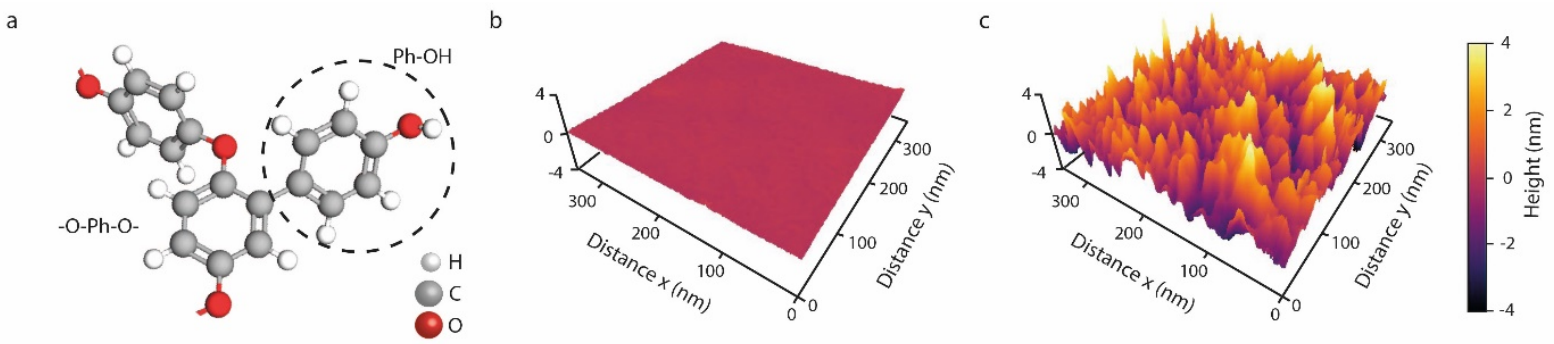

Figure 2. (a) A segment of the PPO structure showing different types of structural units of the PPO layer obtained from XPS analysis. Ph, phenyl group. (b) 3D AFM micrograph of a 350 nm x $350 \mathrm{~nm}$ area on bare HOPG. (c) The same area as in (b) after PPO deposition. The scale bar is common for both AFM images. 


\section{WILEY-VCH}
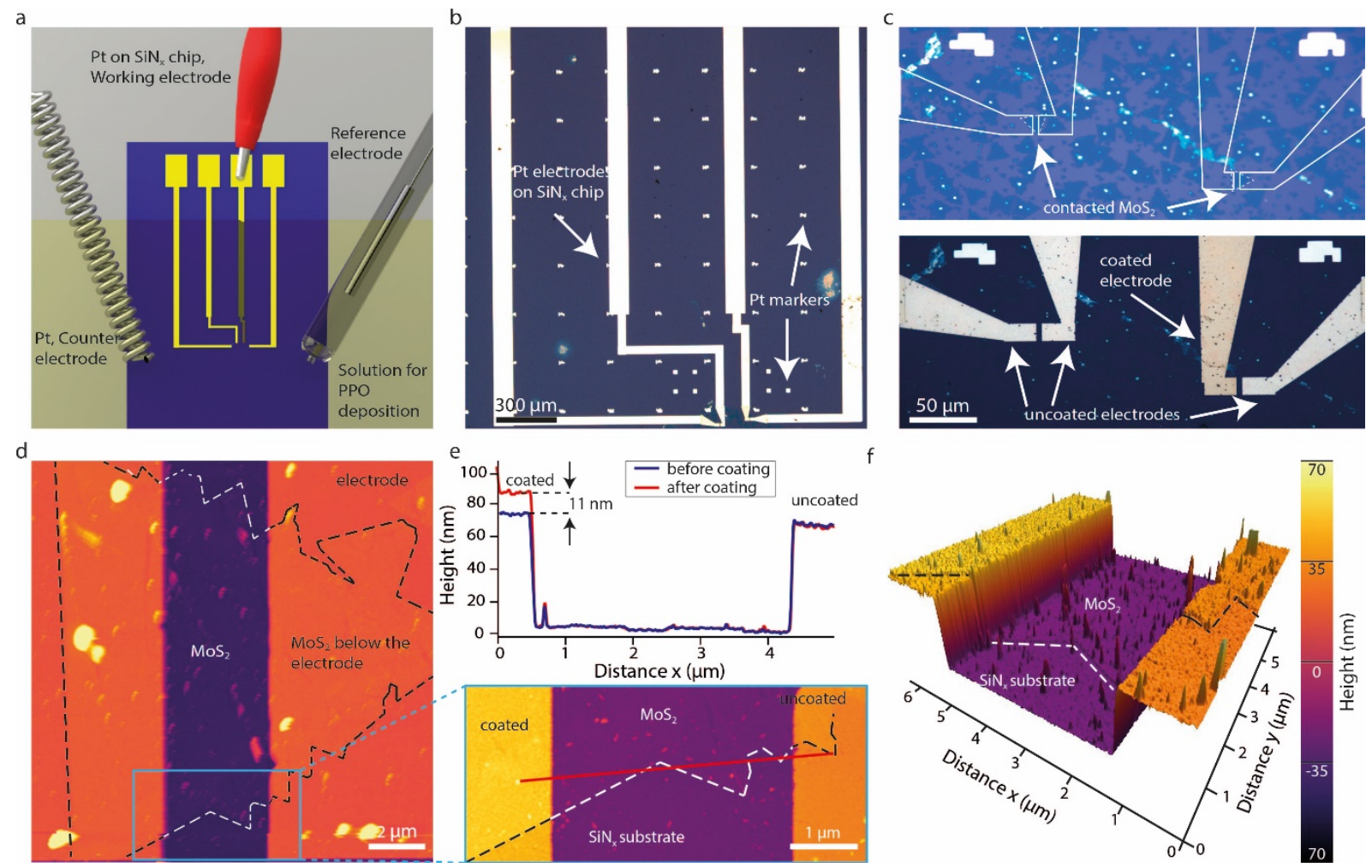

Figure 3. (a) Schematic of the coating setup. The objects are not to scale. (b) An optical micrograph of evaporated Pt electrodes that are contacting $\mathrm{MoS}_{2}$. (c) A close-up view of $\mathrm{MoS}_{2}$ single-layer crystals before (top) and after (bottom) the electrode evaporation and coating. The dashed line indicates the contacted monocrystals, while the solid line denotes the position of the electrodes. Optically, a coated electrode appears slightly darker than the pristine electrode. (d) An AFM image of one of the $\mathrm{MoS}_{2}$ crystals contacted by two evaporated electrodes. The white dashed line indicates the edges of the uncovered part of $\mathrm{MoS}_{2}$ flake, while the black dashed line indicates the parts below the electrodes. The gap between the electrodes is $3.8 \mu \mathrm{m}$. The image was taken before the coating. (e) An AFM image of the area indicated in (d) by a light-blue square (bottom). The cross-section (top) was taken before and after the coating at the place represented by the red solid line (bottom). (f) A 3D representation of the AFM data from the same area as in e (bottom). The scale bar is common for all AFM images. 


\section{WILEY-VCH}
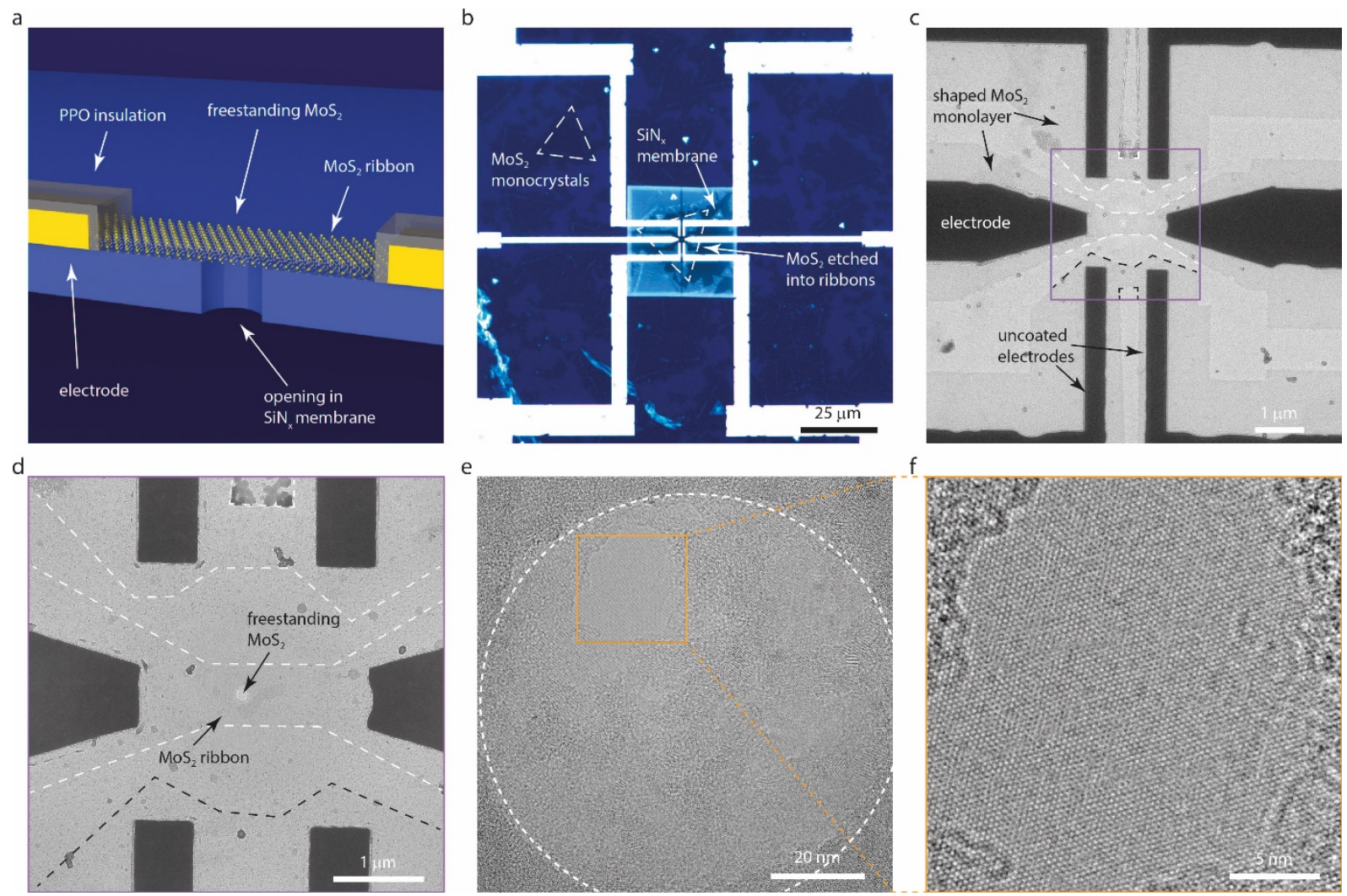

Figure 4 (a) A cross-section schematic of the device. The objects are not to scale. (b) An optical image of a device with a $\mathrm{MoS}_{2}$ monocrystal (indicated with a dashed line) transferred over an aperture in a $\mathrm{SiN}_{\mathrm{x}}$ membrane (light blue square). The $\mathrm{MoS}_{2}$ monocrystal is etched into three ribbons which are then contacted by three pairs of electrodes. (c) A TEM micrograph of the same device after PPO coating. The image is out of focus to emphasize the $\mathrm{MoS}_{2}$ ribbons that, in this condition, appear slightly darker than the rest of the $\mathrm{SiN}_{\mathrm{x}}$ surface. Electrodes contacting the upper and the middle ribbon were coated with PPO. The white dashed lines indicate the edges of the nanoribbons connected to the coated electrodes. The black dashed line indicates the edges of the nanoribbon connected to uncoated electrodes. The PPO coating cannot be seen during TEM imaging since the metallic electrodes are impenetrable for the electron beam. (d) A TEM micrograph of the region denoted by purple square in (c). The arrow indicates the 80 nm-diameter aperture in the center of the ribbon where $\mathrm{MoS}_{2}$ is suspended. (e) HR-TEM image of the freestanding $\mathrm{MoS}_{2}$ part. (f) Zoom-in into a clean area indicated by orange square in (e), revealing the lattice of the freestanding $\mathrm{MoS}_{2}$. 


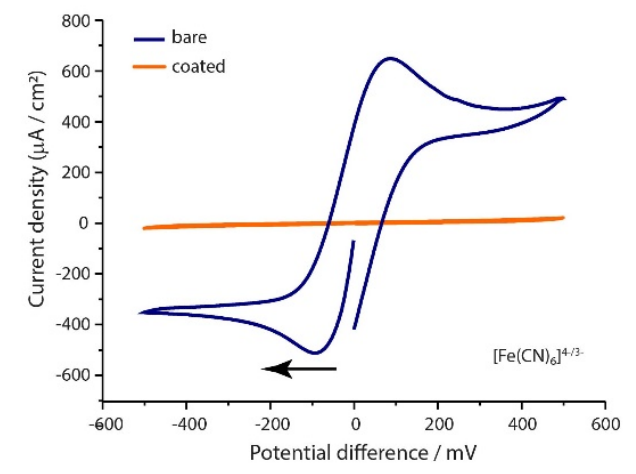

Figure 5. Two-electrode system. Current density vs. potential difference applied between the electrodes recorded in $5 \mathrm{mM}\left[\mathrm{Fe}(\mathrm{CN})_{6}\right]^{4-3-}$, with $0.1 \mathrm{M} \mathrm{KNO}_{3}$ as a supporting electrolyte, before and after PPO deposition. The scan rate was $50 \mathrm{mV} \mathrm{s}^{-1}$. The arrow indicates the scan direction. 